PROCEEDINGS OF THE

AMERICAN MATHEMATICAL SOCIETY

Volume 138, Number 11, November 2010, Pages 4025-4035

S 0002-9939(2010)10397-0

Article electronically published on May 17, 2010

\title{
ON THE BARYCENTER OF THE TENT MAP
}

\author{
KUO-CHANG CHEN AND XUN DONG
}

(Communicated by Bryna Kra)

\begin{abstract}
It is well known that the average position or barycenter of generic orbits for the standard tent map is 0.5. Periodic orbits are exceptional orbits in the sense that most of them have barycenters different from 0.5. In this paper we prove that for any positive integer $n$, there exist $n$ distinct periodic orbits for the standard tent map with the same barycenter. We also provide some patterns of periodic orbits with the same barycenter.
\end{abstract}

\section{INTRODUCTION}

Let $T:[0,1] \rightarrow[0,1]$ be the standard (full) tent map defined by $T(x)=1-|2 x-1|$ or

$$
T(x)= \begin{cases}2 x & \text { when } x \in\left[0, \frac{1}{2}\right] \\ 2(1-x) & \text { when } x \in\left[\frac{1}{2}, 1\right] .\end{cases}
$$

This map is often introduced as one of the first examples of chaotic maps in typical textbooks for dynamical systems. Its dynamics exhibit various features that are commonly used to identify chaotic systems (see for instance [1]).

The $n$-th iterate of $T$ is given by

$$
T^{n}(x)= \begin{cases}2^{n}\left(x-\frac{2 m}{2^{n}}\right) & \text { when } x \in\left[\frac{2 m}{2^{n}}, \frac{2 m+1}{2^{n}}\right], \\ 2^{n}\left(\frac{2(m+1)}{2^{n}}-x\right) & \text { when } x \in\left[\frac{2 m+1}{2^{n}}, \frac{2(m+1)}{2^{n}}\right]\end{cases}
$$

for $m \in\left\{0,1, \cdots, 2^{n-1}-1\right\}$. Fixed points of $T^{n}$, or $n$-periodic points of $T$, are clearly of the form $\frac{2 m}{2^{n}-1}$ or $\frac{2 m}{2^{n}+1}$. Given an $n$-periodic point $x \in(0,1)$, the average

$$
\bar{x}=\frac{1}{n}\left(x+T(x)+\cdots+T^{n-1}(x)\right)
$$

over its orbit is clearly the ergodic average (or time average) of $T$ at $x$. It is also called the barycenter, centroid, or mass center of the orbit. By the ergodic average of $f \in L^{1}[0,1]$ at $x \in[0,1]$ we mean the limit value, if it exists, of $\frac{1}{n} \sum_{k=0}^{n-1} f\left(T^{i} x\right)$ as $n \rightarrow \infty$. Since the Lebesgue measure is an ergodic invariant measure, by the Birkhoff ergodic theorem we know that for almost every $x$ (in the sense of Lebesgue)

Received by the editors November 11, 2009 and, in revised form, January 19, 2010.

2010 Mathematics Subject Classification. Primary 37E05; Secondary 37E45.

Key words and phrases. Tent map, periodic points, barycenter.

This work is partially supported by the National Science Council and the National Center for Theoretical Sciences (NCTS) in Taiwan. Part of the work was completed while the second author was a visiting researcher at the Center in 2007. The second author would like to thank the NCTS for its support. We also thank M. Misiurewicz for helpful comments on the first draft and the referee for helpful remarks.

(C)2010 American Mathematical Society Reverts to public domain 28 years from publication 
the mass center of its orbit is 0.5 , the same as the space average of $T$. It is not difficult to prove that the set of all barycenters is $[0,2 / 3]$.

In 2] Misiurewicz noted that two cycles with mirror itineraries have the same barycenter. He then raised an interesting question: Can there be three different cycles with the same barycenter? The answer is affirmative as can easily be verified from the distinct orbits of

$$
\frac{166}{4095}, \frac{202}{4095}, \frac{278}{4095}, \frac{418}{4095}
$$

or the distinct orbits of

$$
\frac{42}{4097}, \frac{54}{4097}, \frac{134}{4097}, \frac{190}{4097} .
$$

The orbits of

$$
\frac{2250}{16385}, \frac{2266}{16385}, \frac{2446}{16385}, \frac{2490}{16385}, \frac{2510}{16385}
$$

are also distinct but with the same barycenter, and so are the orbits of

$$
\frac{1510}{65535}, \frac{1658}{65535}, \frac{2270}{65535}, \frac{3566}{65535}, \frac{3830}{65535}, \frac{3938}{65535} \text {. }
$$

As the denominator increases there are more and more examples of distinct cycles with the same barycenter. The search for $n$ distinct cycles with the same barycenter becomes a challenging task when $n$ is large. In this paper we prove that

Theorem 1.1. For any positive integer $n$, there exist $n$ distinct cycles for the standard tent map with the same barycenter.

As another incentive for our work, we remark here that for baker maps $B(x)=$ $k x \bmod 1$ (in particular the most standard case $k=2$ ) one can very easily check that many periodic orbits share the same barycenter, but intriguingly this is by no means obvious for the tent map.

In section 2 we provide a sufficient condition for two periodic orbits to have the same barycenter. Section 3 is devoted to the proof of Theorem 1.1. Some other patterns of periodic orbits with the same barycenter are given in section 4 Final remarks and related problems are put into section 5 .

\section{Binary REPRESEntations And the trace VeCtor}

In this section we introduce the concept of a trace vector and establish a sufficient condition for periodic orbits of a certain type to have the same barycenter.

Throughout this and the next section we consider $n$-periodic points ( $n$ is not necessarily the minimum period) of the form $x=\frac{2 m}{2^{n}-1}$, because other $n$-periodic points $\frac{2 m}{2^{n}+1}$ can be expressed in the same form $\frac{2 m\left(2^{n}-1\right)}{2^{2 n}-1}$. The numerator $2 m$ of $x$ can be uniquely written as

$$
a_{1} 2^{n-1}+a_{2} 2^{n-2}+\cdots+a_{n-1} 2^{1}
$$

with $a_{i} \in\{0,1\}$ for all $i$. For convenience we add the term $a_{n} 2^{0}$ with coefficient $a_{n}=0$ and write the numerator as a vector in $(\mathbb{Z} / 2 \mathbb{Z})^{n}$ :

$$
2 m=\left[a_{1}, a_{2}, \cdots, a_{n}\right] .
$$

The tent map $T$ acts on the vector as a cyclic left-shift when $a_{1}=0$, while 0 's and 1 's are swapped after a cyclic left-shift when $a_{1}=1$. More precisely, the tent map 
induces a map $T_{*}$ from $\left\{0,1,2, \cdots, 2^{n}-1\right\}$ to $\left\{0,2,4, \cdots, 2^{n-1}-2\right\}$ given by

$$
\begin{aligned}
T_{*}\left[a_{1}, a_{2}, \cdots, a_{n}\right] & = \begin{cases}{\left[a_{2}, a_{3}, \cdots, a_{n}, a_{1}\right]} & \text { if } a_{1}=0, \\
{\left[1-a_{2}, 1-a_{3}, \cdots, 1-a_{n}, 1-a_{1}\right]} & \text { if } a_{1}=1\end{cases} \\
& =\left[a_{2}, a_{3}, \cdots, a_{n}, a_{1}\right]+\left[a_{1}, a_{1}, \cdots, a_{1}, a_{1}\right] \\
& =\left[a_{1}, a_{2}, \cdots, a_{n-1}, a_{n}\right] E F,
\end{aligned}
$$

where $E, F \in M_{n \times n}(\mathbb{Z} / 2 \mathbb{Z})$ :

$$
E=\left[\begin{array}{ccccc}
0 & 0 & \cdots & 0 & 1 \\
1 & 0 & \cdots & 0 & 0 \\
0 & 1 & 0 & \cdots & 0 \\
\vdots & \ddots & \ddots & \ddots & \vdots \\
0 & \cdots & 0 & 1 & 0
\end{array}\right], F=I_{n}+\left[\begin{array}{ccccc}
0 & 0 & \cdots & 0 & 0 \\
0 & 0 & \cdots & 0 & 0 \\
\vdots & \vdots & \ddots & \vdots & \vdots \\
0 & 0 & \cdots & 0 & 0 \\
1 & 1 & \cdots & 1 & 1
\end{array}\right] .
$$

Note that the last entry of $T_{*}\left[a_{1}, a_{2}, \cdots, a_{n}\right]$ is always zero. Therefore,

$$
\begin{aligned}
& T_{*}^{k}\left[a_{1}, a_{2}, \cdots, a_{n}\right] \\
= & {\left[a_{1}, a_{2}, \cdots, a_{n}\right](E F)^{k} } \\
= & {\left[a_{1}, a_{2}, \cdots, a_{n}\right] E^{k}\left(E^{1-k} F E^{k-1}\right)\left(E^{2-k} F E^{k-2}\right) \cdots\left(E^{-1} F E^{1}\right) F } \\
= & {\left[a_{k+1}, \cdots, a_{n}, a_{1}, \cdots, a_{k}\right]+\left[a_{k}, a_{k}, \cdots, a_{k}\right] . }
\end{aligned}
$$

The last identity holds because

$$
\left[a_{1}, a_{2}, \cdots, a_{n}\right] E^{k}=\left[a_{k+1}, \cdots, a_{n}, a_{1}, \cdots, a_{k}\right]
$$

and the collected effect of

$$
\left(E^{1-k} F E^{k-1}\right)\left(E^{2-k} F E^{k-2}\right) \cdots\left(E^{-1} F E^{1}\right) F
$$

amount to adding a vector $[c, c, \cdots, c]$ to $\left[a_{k+1}, \cdots, a_{n}, a_{1}, \cdots, a_{k}\right]$. Since the last entry of $T_{*}^{k}\left[a_{1}, a_{2}, \cdots, a_{n}\right]$ is zero, $c$ must be equal to $a_{k}$.

The orbit of $x$ is encoded in the $n$ by $n$ matrix $A(x) \in M_{n \times n}(\mathbb{Z} / 2 \mathbb{Z})$ :

$$
\begin{aligned}
A(x)= & {\left[\begin{array}{c}
a_{1}, a_{2}, \cdots, a_{n} \\
T_{*}\left[a_{1}, a_{2}, \cdots, a_{n}\right] \\
T_{*}^{2}\left[a_{1}, a_{2}, \cdots, a_{n}\right] \\
\vdots \\
T_{*}^{n-1}\left[a_{1}, a_{2}, \cdots, a_{n}\right]
\end{array}\right] } \\
= & {\left[\begin{array}{ccccc}
a_{1} & a_{2} & \cdots & a_{n-1} & a_{n} \\
a_{2} & a_{3} & \cdots & a_{n} & a_{1} \\
\vdots & \vdots & \ddots & \vdots & \vdots \\
a_{n} & a_{1} & \cdots & a_{n-2} & a_{n-1}
\end{array}\right]+\left[\begin{array}{cccc}
a_{n} & a_{n} & \cdots & a_{n} \\
a_{1} & a_{1} & \cdots & a_{1} \\
\vdots & \vdots & \ddots & \vdots \\
a_{n-1} & a_{n-1} & \cdots & a_{n-1}
\end{array}\right] } \\
= & {\left[\alpha, E^{-1} \alpha, \cdots, E^{1-n} \alpha\right]+[E \alpha, E \alpha, \cdots, E \alpha], }
\end{aligned}
$$

where $\alpha=\left[a_{1}, a_{2}, \cdots, a_{n}\right]^{T}$. Let $\beta=\alpha+E \alpha$ and

$$
C_{k}=I+E^{-1}+\cdots+E^{-k} \text {. }
$$

Then

$$
E^{-k} \alpha+E \alpha=\left(I+E^{-1}+\cdots+E^{-k}\right)(\alpha+E \alpha)=C_{k} \beta .
$$


Therefore

$$
A(x)=\left[\beta, C_{1} \beta, \cdots, C_{n-1} \beta\right] .
$$

The last column of $A(x)$ is zero. Since all entries of the matrix $C_{n-1}$ are 1 , we conclude that the number of 1's in $\beta$ must be even. Also note that the first column $\beta=\alpha+E \alpha$ of $A(x)$ is exactly the itinerary of $x=\frac{2 m}{2^{n}-1}$ (this follows from the definition of $A(x))$; that is, $\beta_{i}=\chi_{\left[\frac{1}{2}, 1\right]}\left(T^{i-1}(x)\right)$ for each $i$.

Note that $\frac{2 m}{2^{n}-1}$ and $\frac{2 k}{2^{n}-1}$ cannot have the same itinerary unless $m=k$. Since there are $2^{n-1}$ vectors of the form $\left[a_{1}, \cdots, a_{n-1}, 0\right]$ and $2^{n-1}$ possible itineraries $\beta$ (which must have an even number of 1's), the correspondence between $x=\frac{2 m}{2^{n}-1}$ and $\beta$ is necessarily bijective.

Let $s_{j}(\beta)=s_{j}$ be the number of 1's in the $j$-th column $C_{j-1} \beta$ of $A(x)$. We call

$$
s(\beta)=\left(s_{1}, s_{2}, \cdots, s_{n}\right) \in \mathbb{Z}^{n}
$$

the trace vector of $x$. If follows easily from (2.1) that $s_{n}=0$ and each $s_{j}$ is even. Since

$C_{n-k-1} \beta=E^{1-(n-k)} \alpha+E \alpha=E^{1+k} \alpha+E \alpha=E^{k}\left(E \alpha+E^{1-k} \alpha\right)=E^{k} C_{k-1} \beta$,

we also have

$$
s_{n-k}=s_{k} \text { for } k=1, \cdots, n-1 .
$$

Write $A(x)=\left[b_{i j}\right]$ with indices $i$ and $j$ ranging from 1 to $n$; then $b_{i j}=\sum_{r=0}^{j-1} \beta_{i+r}$ $\in \mathbb{Z} / 2 \mathbb{Z}$. It is understood that the subindex $i+r$ of each $\beta_{i+r}$ is in $\mathbb{Z} / n \mathbb{Z}$. The orbit of $x$ has barycenter

$$
\bar{x}=\frac{1}{\left(2^{n}-1\right) n} \sum_{i, j=1}^{n} 2^{n-j} b_{i j}=\frac{1}{\left(2^{n}-1\right) n} \sum_{j=1}^{n} 2^{n-j} s_{j} .
$$

Thus for two n-periodic points $x=\frac{2 m}{2^{n}-1}$ and $y=\frac{2 k}{2^{n}-1}$, a sufficient condition for $\bar{x}=\bar{y}$ is that they have the same trace vector.

Let $\beta$ and $\gamma$ denote respectively the itineraries of $n$-periodic points $x$ and $y$. As explained earlier, $x=y$ if and only if $\beta=\gamma$. It follows that $x$ and $y$ are in the same cycle if and only if $\gamma=E^{k} \beta$ for some $k$, in which case we will say that $\beta$ and $\gamma$ are equivalent.

Let

$$
D=\left[\begin{array}{ccccc}
0 & \cdots & 0 & 0 & 1 \\
0 & \cdots & 0 & 1 & 0 \\
\vdots & & & & \vdots \\
0 & 1 & 0 & \cdots & 0 \\
1 & 0 & 0 & \cdots & 0
\end{array}\right]
$$

Then $D$ and $E$ generate a dihedral group of order $2 n$ with relations

$$
E^{n}=D^{2}=(E D)^{2}=I_{n} .
$$

Observe that

$$
C_{k} E=E C_{k}, \quad C_{k} D=D E^{k} C_{k}
$$

for all $k$

If $\gamma=E \beta$, then $C_{k} \gamma=C_{k} E \beta=E C_{k} \beta$ for all $k$. Thus $A(x)$ and $A(y)$ have the same trace vector as expected. 
If $\gamma=D \beta$, then $C_{k} \gamma=C_{k} D \beta=D E^{k} C_{k} \beta$ for all $k$. Thus $A(x)$ and $A(y)$ have the same trace vector. Note that $\gamma$ is the mirror image of $\beta$. In this case $x$ and $y$ belong to different cycles except when $\beta=D E^{k} \beta$ for some $k$. For instance, the point $x=\frac{26}{127}$ has itinerary

$$
\beta=[0,0,1,0,1,1,1]^{T} .
$$

The itinerary of $y=\frac{88}{127}$ is $\beta$-reversed. These two points $x$ and $y$ belong to two different cycles with the same barycenter $\frac{72}{127}$.

One might attempt to look for other permutation matrices $P$ with the nice property that $s(\beta)=s(P \beta)$ for all itineraries $\beta$. Unfortunately there are no such permutation matrices other than those in the dihedral group generated by $D$ and $E$. In order to find three or more different cycles with the same barycenter, we need more explicit constructions, as to be shown in the next section.

\section{A FAMily OF CYClES With THE SAME BARYCENTER}

In order to construct $n$ distinct cycles with the same barycenter, we will find $n$ $(8 n)$-periodic points such that their itineraries $\beta^{(1)}, \cdots, \beta^{(n)}$ have the same trace vector, yet $\beta^{(i)}$ and $\beta^{(j)}$ are non-equivalent when $i \neq j$. The " $n$ " in this section is not to be confused with the " $n$ " in the previous section, which is replaced by " $8 n$ " here for convenience.

For each $1 \leq m \leq n$, let

$$
\beta^{(m)}=\left[\beta_{1}^{(m)}, \beta_{2}^{(m)}, \cdots, \beta_{8 n}^{(m)}\right]^{T}
$$

be defined by the following conditions:

$$
\begin{aligned}
\beta_{i}^{(m)}+\beta_{4 n+i}^{(m)} & =1 \text { for } i=1,2, \cdots, 4 n \\
\beta_{i}^{(m)} & = \begin{cases}1 & \text { if } i \in\{2 m, 2 m+1\} \\
0 & \text { if } i \in\{1,2, \cdots, 4 n\} \backslash\{2 m, 2 m+1\} .\end{cases}
\end{aligned}
$$

It is easy to see that these conditions uniquely determine $\beta^{(m)}$. It is understood that the subindex $i$ of each $\beta_{i}^{(m)}$ is in $\mathbb{Z} / 8 n \mathbb{Z}$. For instance, when $n=3$, the $\beta^{(m)}$ 's are

$$
\begin{aligned}
& \beta^{(1)}=[0,1|1,0| 0,0|0,0| 0,0|0,0| 1,0|0,1| 1,1|1,1| 1,1 \mid 1,1]^{T}, \\
& \beta^{(2)}=[0,0|0,1| 1,0|0,0| 0,0|0,0| 1,1|1,0| 0,1|1,1| 1,1 \mid 1,1]^{T}, \\
& \beta^{(3)}=[0,0|0,0| 0,1|1,0| 0,0|0,0| 1,1|1,1| 1,0|0,1| 1,1 \mid 1,1]^{T} .
\end{aligned}
$$

The vertical bars are inserted in the vectors above to improve readability.

By (2.2), we only need to show that $s_{k}\left(\beta^{(m)}\right)$ is independent of $m$ for $1 \leq k \leq 4 n$ because of the symmetry of the trace vector.

If $k$ is odd, then for each $i \in \mathbb{Z} / 8 n \mathbb{Z}$ we have

$$
b_{i, k}^{(m)}+b_{4 n+i, k}^{(m)}=\sum_{j=0}^{k-1} \beta_{i+j}^{(m)}+\sum_{j=0}^{k-1} \beta_{4 n+i+j}^{(m)}=k \equiv 1 \quad \bmod 2 .
$$

Hence there are as many 1's as 0 's in $\left[b_{1, k}^{(m)} b_{2, k}^{(m)} \cdots b_{8 n, k}^{(m)}\right]^{T}$, and therefore $s_{k}\left(\beta^{(m)}\right)$ $=4 n$ when $k$ is odd. 
Next we note that

$$
b_{i, 4 n}^{(m)}=\sum_{j=0}^{4 n-1} \beta_{i+j}^{(m)}=b_{i+1,4 n}^{(m)}+\beta_{i}^{(m)}-\beta_{4 n+i}^{(m)}=b_{i+1,4 n}^{(m)}+1 .
$$

Hence there are as many 1's as 0 's in $\left[b_{1,4 n}^{(m)} b_{2,4 n}^{(m)} \cdots b_{8 n, 4 n}^{(m)}\right]^{T}$, and therefore $s_{4 n}\left(\beta^{(m)}\right)$ $=4 n$.

We now assume that $k=2 r$ where $1 \leq r<2 n$. Note that

$$
\begin{aligned}
& \beta_{2 i-1}^{(m)}+\beta_{2 i}^{(m)}= \begin{cases}1 & \text { when } i \in\{m, m+1,2 n+m, 2 n+m+1\}, \\
0 & \text { otherwise, }\end{cases} \\
& \beta_{2 i}^{(m)}+\beta_{2 i+1}^{(m)}= \begin{cases}1 & \text { when } i \in\{0,2 n\}, \\
0 & \text { otherwise. }\end{cases}
\end{aligned}
$$

It follows that

$$
\begin{aligned}
b_{2 i-1,2 r}^{(m)} & =\sum_{j=0}^{r-1}\left(\beta_{2 i+2 j-1}^{(m)}+\beta_{2 i+2 j}^{(m)}\right) \\
& = \begin{cases}1 & \text { if } i \in\{m+1, m-r+1,2 n+m+1,2 n+m-r+1\}, \\
0 & \text { otherwise }\end{cases}
\end{aligned}
$$

and

$$
\begin{aligned}
b_{2 i, 2 r}^{(m)} & =\sum_{j=0}^{r-1}\left(\beta_{2 i+2 j}^{(m)}+\beta_{2 i+2 j+1}^{(m)}\right) \\
& = \begin{cases}1 & \text { if either }\{0,1\} \text { or }\{4 n, 4 n+1\} \subset\{2 i, 2 i+1, \cdots, 2 i+2 r-1\}, \\
0 & \text { otherwise. }\end{cases}
\end{aligned}
$$

There are four 1's in the first case and $2 r$ 1's in the second case. Hence $s_{k}\left(\beta^{(m)}\right)=$ $2 r+4=k+4$.

It remains to show that if $i \neq j$, then $\beta^{(i)}$ and $\beta^{(j)}$ are not equivalent; that is, $\beta^{(i)}$ cannot be obtained from $\beta^{(j)}$ by a cyclic rotation. This follows from the fact that if we write $\beta^{(m)}$ around a circle, then the length of the longest substring of consecutive 0's is $4 n-2 m-1$. Thus the $\beta^{(m)}$ 's are itineraries of points in $n$ distinct cycles with the same barycenter. The proof of Theorem 1.1 is now concluded.

Remark 3.1. It is easy to see that the definition of $\beta^{(m)}$ can be extended to all $1 \leq m<2 n$. In fact, all these $2 n-1$ itineraries are pairwise non-equivalent. Moreover there is another itinerary with the same trace vector, making a total of $2 n$ distinct $(8 n)$-cycles with the same barycenter.

Replacing $2 n$ by $n$, we may construct $n$ distinct $(4 n)$-cycles with the same barycenter as follows. For each $0 \leq m<n$, let

$$
\gamma^{(m)}=\left[\gamma_{1}^{(m)}, \gamma_{2}^{(m)}, \cdots, \gamma_{4 n}^{(m)}\right]^{T} \in(\mathbb{Z} / 2 \mathbb{Z})^{4 n},
$$

where $\gamma_{i}^{(m)}+\gamma_{2 n+i}^{(m)}=1$ for $i=1,2, \cdots, 2 n$. For $m=0$ define

$$
\gamma_{i}^{(0)}= \begin{cases}1 & \text { if } i \in\{1,3\}, \\ 0 & \text { if } i \in\{1,2, \cdots, 2 n\} \backslash\{1,3\},\end{cases}
$$

and for $1 \leq m<n$ define

$$
\gamma_{i}^{(m)}= \begin{cases}1 & \text { if } i \in\{2 m, 2 m+1\} \\ 0 & \text { if } i \in\{1,2, \cdots, 2 n\} \backslash\{2 m, 2 m+1\} .\end{cases}
$$


Then a similar calculation shows that $s\left(\gamma^{(m)}\right)$ is independent of $m$, with $s_{2 r-1}=$ $s_{2 n}=2 n$ and $s_{2 r}=2 r+4$ for $r=1, \cdots, n$. It is also not hard to verify that if $i \neq j$, then $\gamma^{(i)}$ and $\gamma^{(j)}$ are not equivalent. Therefore we obtain $n$ distinct $(4 n)$-cycles with the same barycenter.

Remark 3.2. Let $x^{(m)}$ denote the $(4 n)$-periodic point with itinerary $\gamma^{(m)}$ for $0 \leq$ $m<n$. Then it can be calculated that

$$
\begin{aligned}
x^{(0)} & =\frac{2}{3\left(2^{2 n}+1\right)}+\frac{3 \cdot 2^{4 n-2}-2^{2 n-2}}{2^{4 n}-1} \\
x^{(m)} & =\frac{2}{3\left(2^{2 n}+1\right)}+\frac{2^{2 n-2 m}}{2^{2 n}-1} \quad \text { for } m=1, \cdots, n-1 .
\end{aligned}
$$

Using equations (2.2), (2.3) and the common trace vector of $x^{(m)}$ 's, one can calculate the common mass center of their orbits:

$$
\begin{aligned}
\bar{x} & =\frac{1}{4 n\left(2^{4 n}-1\right)}\left[2^{2 n} s_{2 n}+\sum_{\substack{j=1 \\
j \text { is odd }}}^{4 n-1} 2^{4 n-j} s_{j}+\sum_{\substack{j=2 \\
j \text { is even }}}^{2 n-2} 2^{4 n-j} s_{j}+\sum_{\substack{j=2 n+2 \\
j \text { is even }}}^{4 n-2} 2^{4 n-j} s_{j}\right] \\
& =\frac{1}{4 n\left(2^{4 n}-1\right)}\left[2 n\left(2^{2 n}+\sum_{r=0}^{2 n-1} 2^{2 r+1}\right)\right. \\
& =\frac{1}{3}+\frac{5}{9 n}-\frac{13}{9 n\left(2^{2 n}+1\right)}-\frac{2}{n\left(2^{4 n}-1\right)} .
\end{aligned}
$$

If we replace $n$ by $2 n$ in these formulas, then we obtain the $(8 n)$-periodic points with itineraries $\beta^{(m)}$ and the common barycenter of their orbits.

\section{Further EXAmples of CyCles With the SAME BARYCEnter}

There are several patterns of itineraries that can be suitably permuted without altering their trace vectors. We will discuss one of them to which $\beta^{(1)}$ and $\gamma^{(1)}$ in section 3 belong.

To simplify our notation, we will denote itineraries by row vectors instead of column vectors in this section. For any string $\mathbf{u}=\left[u_{1}, \cdots, u_{k}\right]$ consisting of 0 's and 1 's, $\mathbf{u}^{-1}$ denotes the string obtained by reversing the order of the $u_{i}$ 's in $\mathbf{u}$, and $|\mathbf{u}|$ denotes the sum of the $u_{i}$ 's over $\mathbb{Z} / 2 \mathbb{Z}$. All itineraries are treated as cyclic vectors.

Proposition 4.1. Let $\beta$ be an itinerary of the form

$$
\beta=\left[0, \mathbf{p}, 0, \mathbf{q}, 1, \mathbf{p}^{-1}, 1, \mathbf{r}\right],
$$

where $\mathbf{p}=\left[p_{1}, \cdots, p_{j}\right], \mathbf{q}=\left[q_{1}, \cdots, q_{k}\right]$ and $\mathbf{r}=\left[r_{1}, \cdots, r_{k}\right]$ are subject to the conditions

$$
\mathbf{q}=\mathbf{q}^{-1}, \quad \mathbf{r}=\mathbf{r}^{-1}, \quad|\mathbf{q}|=|\mathbf{r}| .
$$

Then the trace vector of $\beta$ does not change if $\mathbf{p}$ and $\mathbf{p}^{-1}$ are switched; that is, $s(\beta)=s(\gamma)$, where

$$
\gamma=\left[0, \mathbf{p}^{-1}, 0, \mathbf{q}, 1, \mathbf{p}, 1, \mathbf{r}\right]
$$


Note that the presence of the two 0's and 1's implies that $\gamma$ is in general not in the orbit of $\beta$ under the action of the dihedral group (2.4). We will call the two 0's and 1's the four corners of $\beta$ and $\gamma$.

Proof. Let $n=2 j+2 k+4$ denote the length of $\beta$. By (2.2), we only need to show that $s_{l}(\beta)=s_{l}(\gamma)$ for $1 \leq l \leq n / 2$. Let us fix such an $l$. Define

$$
\begin{array}{ll}
\beta^{\prime}=\left[0, \mathbf{p}^{-1}, 0, \mathbf{r}, 1, \mathbf{p}, 1, \mathbf{q}\right], & \gamma^{\prime}=\left[0, \mathbf{p}, 0, \mathbf{r}, 1, \mathbf{p}^{-1}, 1, \mathbf{q}\right], \\
\beta^{\prime \prime}=\left[1, \mathbf{p}, 1, \mathbf{q}, 0, \mathbf{p}^{-1}, 0, \mathbf{r}\right], & \gamma^{\prime \prime}=\left[1, \mathbf{p}^{-1}, 1, \mathbf{q}, 0, \mathbf{p}, 0, \mathbf{r}\right], \\
\beta^{\prime \prime \prime}=\left[1, \mathbf{p}^{-1}, 1, \mathbf{r}, 0, \mathbf{p}, 0, \mathbf{q}\right], & \gamma^{\prime \prime \prime}=\left[1, \mathbf{p}, 1, \mathbf{r}, 0, \mathbf{p}^{-1}, 0, \mathbf{q}\right] .
\end{array}
$$

Then $\beta^{\prime}, \beta^{\prime \prime}$ and $\beta^{\prime \prime \prime}$ are in the same orbit of $\beta$ under the action of the dihedral group (2.4), so they all have the same trace vector. Similarly $\gamma^{\prime}, \gamma^{\prime \prime}$ and $\gamma^{\prime \prime \prime}$ are in the same orbit of $\gamma$, so they all have the same trace vector as well. For a cyclic vector $\mathbf{x}=\left[x_{1}, \cdots, x_{n}\right]$, let $\widehat{\mathbf{x}}=\left[x_{i}, \cdots, x_{i+l-1}\right]$, where it is understood that the subindices are in $\mathbb{Z} / n \mathbb{Z}$. Since $l \leq n / 2$, the substring $\widehat{\beta}$ covers at most two corners of $\beta$.

Case 1: $\widehat{\beta}$ covers zero corners. If $\widehat{\beta}$ covers nothing of $\mathbf{p}$ and $\mathbf{p}^{-1}$, then note that $\gamma, \gamma^{\prime}, \gamma^{\prime \prime}$ and $\gamma^{\prime \prime \prime}$ are obtained from $\beta, \beta^{\prime}, \beta^{\prime \prime}$ and $\beta^{\prime \prime \prime}$ by switching $\mathbf{p}$ and $\mathbf{p}^{-1}$. If $\widehat{\beta}$ covers nothing of $\mathbf{q}$ and $\mathbf{r}$, then note that $\gamma, \gamma^{\prime}, \gamma^{\prime \prime}$ and $\gamma^{\prime \prime \prime}$ are obtained from $\beta^{\prime}, \beta, \beta^{\prime \prime \prime}$ and $\beta^{\prime \prime}$ by switching $\mathbf{q}$ and $\mathbf{r}$. In either case we have $\left\{|\widehat{\beta}|,\left|\widehat{\beta^{\prime}}\right|,\left|\widehat{\beta^{\prime \prime}}\right|,\left|\widehat{\beta^{\prime \prime \prime}}\right|\right\}$ and $\left\{|\widehat{\gamma}|,\left|\widehat{\gamma^{\prime}}\right|,\left|\widehat{\gamma^{\prime \prime}}\right|,\left|\widehat{\gamma^{\prime \prime \prime}}\right|\right\}$ are the same as multisets.

Case 2: $\widehat{\beta}$ covers one corner. Notice that if we switch 0 and 1 in the four corners, then $\beta$ becomes $\beta^{\prime \prime}$ and $\beta^{\prime}$ becomes $\beta^{\prime \prime \prime}$. Therefore as multisets $\left\{|\widehat{\beta}|,\left|\widehat{\beta^{\prime}}\right|,\left|\widehat{\beta^{\prime \prime}}\right|,\left|\widehat{\beta^{\prime \prime \prime}}\right|\right\}$ $=\{0,0,1,1\}$. For a similar reason we also have $\left\{|\widehat{\gamma}|,\left|\widehat{\gamma^{\prime}}\right|,\left|\widehat{\gamma^{\prime \prime}}\right|,\left|\widehat{\gamma^{\prime \prime \prime}}\right|\right\}=\{0,0,1,1\}$.

Case 3: $\widehat{\beta}$ covers two corners. The argument here is similar to Case 1 . If $\widehat{\beta}$ covers a complete $\mathbf{p}$ (or $\mathbf{p}^{-1}$ ), then it covers nothing of $\mathbf{p}^{-1}$ (or $\mathbf{p}$ ) since $l \leq n / 2$. In this case switch $\mathbf{p}$ and $\mathbf{p}^{-1}$. If $\widehat{\beta}$ covers a complete $\mathbf{q}$ (or $\mathbf{r}$ ), then it covers nothing of $\mathbf{r}$ (or $\mathbf{q}$ ). In this case switch $\mathbf{q}$ and $\mathbf{r}$. Since $\left|\mathbf{p}^{-1}\right|=|\mathbf{p}|$ and $|\mathbf{q}|=|\mathbf{r}|$, in either case we have $\left\{|\widehat{\beta}|,\left|\widehat{\beta^{\prime}}\right|,\left|\widehat{\beta^{\prime \prime}}\right|,\left|\widehat{\beta^{\prime \prime \prime}}\right|\right\}=\left\{|\widehat{\gamma}|,\left|\widehat{\gamma^{\prime}}\right|,\left|\widehat{\gamma^{\prime \prime}}\right|,\left|\widehat{\gamma^{\prime \prime \prime}}\right|\right\}$.

Now let $i$ run through $\{1, \cdots, n\}$. Then we have $4 s_{l}(\beta)=4 s_{l}(\gamma)$.

Remark 4.2. The same arguments can be applied to itineraries of the form

$$
\beta=\left[0, \mathbf{p}, 1, \mathbf{q}, 0, \mathbf{p}^{-1}, 1, \mathbf{r}\right],
$$

where $\mathbf{p}=\left[p_{1}, \cdots, p_{j}\right], \mathbf{q}=\left[q_{1}, \cdots, q_{k}\right]$ and $\mathbf{r}=\left[r_{1}, \cdots, r_{k}\right]$ are subject to conditions (4.1).

Example 4.3. Both the $\beta^{(1)}$ in (3.1) and the $\gamma^{(1)}$ in (3.3) have the structure described in Proposition 4.1. For instance, the $\beta^{(1)}$ in (3.2) as a cyclic vector can be written as

$$
\beta^{(1)}=[0, \underbrace{1,0}_{\mathbf{p}}, 0, \underbrace{1,1,1,1,1,1,1,1}_{\mathbf{q}}, 1, \underbrace{0,1}_{\mathbf{p}^{-1}}, 1, \underbrace{0,0,0,0,0,0,0,0}_{\mathbf{r}}] .
$$

By Proposition 4.1, the itinerary

$$
\beta^{(0)}=[0, \underbrace{0,1}_{\mathbf{p}^{-1}}, 0, \underbrace{1,1,1,1,1,1,1,1}_{\mathbf{q}}, 1, \underbrace{1,0}_{\mathbf{p}}, 1, \underbrace{0,0,0,0,0,0,0,0}_{\mathbf{r}}]
$$

has the same trace vector as $\beta^{(1)}$. Their trace vector is

$$
(12,6,12,8,12,10,12,12,12,14,12,12,12,14,12,12,12,10,12,8,12,6,12,0) .
$$


Proposition 4.1 also gives another reason why the $\gamma^{(0)}$ and $\gamma^{(1)}$ in (3.3) have the same trace vector.

Example 4.4. Ideally, one would hope to have itineraries where Proposition 4.1 could be applied multiple times to produce many distinct cycles. Here we present such an interesting example for the case $n=20$. Unfortunately it is unclear how to generalize it to general $n$. Let

$$
\beta^{(0)}=[0, \underbrace{0,0,0,1,1,0}_{\mathbf{p}}, 0, \underbrace{0,0}_{\mathbf{q}}, 1, \underbrace{0,1,1,0,0,0}_{\mathbf{p}^{-1}}, 1, \underbrace{1,1}_{\mathbf{r}}] .
$$

After reversing the $p_{i}$ 's and shifting the cyclic vector, the new itinerary $\beta^{(1)}$ also has the pattern studied in Proposition 4.1. By repeating this process, we obtain

$$
\begin{aligned}
& \beta^{(1)}=[0, \underbrace{1,0,0}_{\mathbf{p}}, 0, \underbrace{1,1,0,1,1}_{\mathbf{q}}, 1, \underbrace{0,0,1}_{\mathbf{p}^{-1}}, 1, \underbrace{0,0,0,0,0}_{\mathbf{r}}], \\
& \beta^{(2)}=[0, \underbrace{1,0}_{\mathbf{p}}, 0, \underbrace{0,0,0,0,0,0}_{\mathbf{q}}, 1, \underbrace{\underbrace{0,1}_{1}}_{\mathbf{p}^{-1}}, 1, \underbrace{0,1,1,1,1,0}_{\mathbf{r}}], \\
& \beta^{(3)}=[0, \underbrace{0,0,0,1,1}_{\mathbf{p}}, 0, \underbrace{1,0,1}_{\mathbf{q}}, 1, \underbrace{1,1,0,0,0}_{\mathbf{p}^{-1}}, 1, \underbrace{0,0,0}_{\mathbf{r}}] .
\end{aligned}
$$

We resume $\beta^{(0)}$ by reversing the $p_{i}$ 's in $\beta^{(3)}$. It is easy to see that these itineraries and their mirror itineraries are all different, yielding eight distinct cycles with the same barycenter. In the order of $\beta^{(0)}, \cdots, \beta^{(3)}$ followed by their mirror itineraries, they correspond respectively to

$$
\frac{33658}{1048575}, \frac{504896}{1048575}, \frac{523412}{1048575}, \frac{39664}{1048575}, \frac{776224}{1048575}, \frac{17852}{1048575}, \frac{337916}{1048575}, \frac{125728}{1048575} .
$$

Their common trace vector is

$$
(8,8,8,10,10,10,12,12,12,12,12,12,12,10,10,10,8,8,8,0)
$$

and their common barycenter is $\frac{2170804}{5242875} \approx 0.414048$.

Example 4.5. There are patterns of itineraries that allow many more distinct cycles with the same barycenters than the one constructed in section 3 . However it is unclear how to generalize these patterns. The following itineraries along with their mirror itineraries correspond to 16 cycles of period 24 with the same barycenter:

$$
\begin{aligned}
& \beta^{(0)}=[0,0,0,0,0,0,1,1,1,0,1,0,1,0,0,1,1,1,0,1,1,1,0,1] \\
& \beta^{(1)}=[0,0,0,0,0,1,1,0,1,1,0,1,1,1,1,1,0,1,0,1,0,0,0,1] \\
& \beta^{(2)}=[0,0,0,0,0,1,1,0,1,1,1,0,1,1,0,0,1,0,0,1,1,1,0,1] \\
& \beta^{(3)}=[0,0,0,0,0,1,1,1,1,0,1,0,0,0,1,1,1,0,1,1,0,1,0,1] \\
& \beta^{(4)}=[0,0,0,0,0,1,0,1,0,0,0,1,1,1,1,1,0,1,1,0,1,1,0,1] \\
& \beta^{(5)}=[0,0,0,0,1,1,0,0,0,1,1,1,0,1,1,1,0,1,0,1,1,0,0,1] \\
& \beta^{(6)}=[0,0,0,0,1,1,0,1,1,0,0,1,0,1,1,1,0,1,0,0,0,1,1,1] \\
& \beta^{(7)}=[0,0,0,0,1,0,1,1,0,1,1,0,0,0,1,1,0,1,1,1,1,0,0,1] .
\end{aligned}
$$


From $\beta^{(0)}$ to $\beta^{(7)}$, they are respectively itineraries of

$\frac{183958}{16777215}, \frac{300446}{16777215}, \frac{309014}{16777215}, \frac{343334}{16777215}$,
$\frac{398774}{16777215}, \frac{547438}{16777215}, \frac{596602}{16777215}, \frac{900526}{16777215}$.

The mirror itineraries of $\beta^{(0)}, \cdots, \beta^{(7)}$ correspond respectively to

$\frac{13821568}{16777215}, \frac{15946304}{16777215}, \frac{13752896}{16777215}, \frac{13203776}{16777215}$,
$\frac{14373056}{16777215}, \frac{15512608}{16777215}, \frac{12366112}{16777215}, \frac{15432544}{16777215}$.

Their common trace vector is

$(12,12,10,14,12,12,12,10,14,12,12,16,12,12,14,10,12,12,12,14,10,12,12,0)$, and their common barycenter is $\frac{24897599}{50331645} \approx 0.494671$.

Example 4.6. The criterion in section 2 for two distinct cycles to have the same barycenter is sufficient but not necessary. For example, the fractions $\frac{5414}{131071}$ and $\frac{10090}{131071}$ are points of period 17 with itineraries

$$
\begin{aligned}
& \beta=[0,0,0,0,1,1,1,1,1,1,0,1,1,0,1,0,1], \\
& \gamma=[0,0,0,1,1,0,1,0,0,1,1,0,1,1,1,1,1] .
\end{aligned}
$$

Their trace vectors are respectively

$$
\begin{aligned}
& s(\beta)=(10,8,8,8,10,8,6,8,8,6,8,10,8,8,8,10,0), \\
& s(\gamma)=(10,8,8,8,6,14,8,10,10,8,14,6,8,8,8,10,0) .
\end{aligned}
$$

One can easily check that $\frac{1185588}{2228207} \approx 0.532082$ is their common barycenter.

Example 4.7. Cycles of different lengths may have the same barycenter. For instance, the orbit of $\frac{46}{275}$ has length 20 and the orbit of $\frac{1158}{7735}$ has length 24 . One can easily verify that their barycenters are both equal to $\frac{3}{5}$.

We have mentioned in section 1 that, by the Birkhoff ergodic theorem, the barycenter of generic orbits is $\frac{1}{2}$, the space average or integral of the tent map. Periodic orbits are exceptional, but there are actually examples of cycles where the barycenter is also $\frac{1}{2}$. For instance,

$$
\frac{19}{18564}, \frac{17}{14460}, \frac{13}{4820}, \frac{151}{55692}
$$

are all 24-periodic points and $\frac{1}{2}$ is the common barycenter of their orbits.

\section{SOME REMARKS AND RELATED PROBLEMS}

The discussions presented so far are focused on periodic points of the form $\frac{2 m}{2^{n}-1}$. As we mentioned earlier, they are also applicable to periodic points of the form $\frac{2 m}{2^{n}+1}$ because $\frac{2 m}{2^{n}+1}=\frac{2 m\left(2^{n}-1\right)}{2^{2 n}-1}$. We say that an $n$-cycle is of the first type if it is generated by points of the form $\frac{2 m}{2^{n}-1}$ and cannot be reduced to points of the form $\frac{2 m}{2^{n}+1}$, and it is of the second type if otherwise. Two of the examples in the introduction are of the second type.

Let $c(n)$ denote the maximum number of distinct first type $n$-cycles with the same barycenter and let $d(n)$ denote the same number for second type $n$-cycles. An exhaustive search by computer shows that $c(n)$ and $d(n)$ are: 


\begin{tabular}{c|c|c|c|c|c|c|c|c|c|c|c|c|c}
$n$ & $\leq 6$ & 7 & 8 & 9 & 10 & 11 & 12 & 13 & 14 & 15 & 16 & 17 & 18 \\
\hline$c(n)$ & 1 & 2 & 2 & 2 & 2 & 2 & 4 & 4 & 4 & 4 & 6 & 6 & 6 \\
\hline$d(n)$ & 1 & 2 & 2 & 2 & 2 & 2 & 4 & 4 & 5 & 4 & 4 & 4 & 8 \\
$n$ & 19 & 20 & 21 & 22 & 23 & 24 & 25 & 26 & 27 & 28 & 29 & 30 & 31 \\
\hline$c(n)$ & 8 & 8 & 8 & 8 & 8 & 16 & 10 & 10 & 14 & 38 & 16 & 24 & 10 \\
\hline$d(n)$ & 4 & 8 & 8 & 6 & 8 & 10 & 8 & 10 & 14 & 12 & 16 & 24 & 10
\end{tabular}

Let $e(n)$ be the maximum number of distinct $n$-cycles of either type with the same barycenter. It turns out that $e(n)=\max \{c(n), d(n)\}$ for $n \leq 31$. Also note that in our notation $n$-cycles include all cycles whose lengths are divisors of $n$. If we only consider cycles whose lengths are exactly $n$, then the values of $c(n), d(n)$ and $e(n)$ remain the same for $n \leq 31$. We don't know if this is true for all $n$.

Another interesting question about cycles with the same barycenter is how fast $e(n)$ grows. From section 3 we know that $e(4 n) \geq n$. Is $e(n)$ of order $O(n)$ as $n$ goes to infinity? If so, what are the values of $\liminf _{n \rightarrow \infty} \frac{e(n)}{n}$ and $\lim _{\sup _{n \rightarrow \infty}} \frac{e(n)}{n}$ ? Intuitively $e(n)$ grows faster on composite numbers because itineraries of $n$-cycles with prime $n$ seem to admit fewer possible trace-invariant permutations. Note that the values of $e(29)$ and $e(31)$ are respectively less than half of $e(28)$ and $e(30)$. Each of $e(17)$ and $e(19)$ is attained at only one example where not all of the distinct cycles have the same trace vector. These observations suggest that $e(n)$ grows much slower on prime numbers.

\section{REFERENCES}

1. A. Katok; B. Hasselblatt; Introduction to the modern theory of dynamical systems. Encyclopedia of Mathematics and its Applications, 54. Cambridge University Press, 1995. MR1326374 (96c:58055)

2. M. Misiurewicz; Rotation theory. Proceedings of the RIMS Workshop on Dynamical Systems and Applications, 2006. Available at http://www.math.kyoto-u.ac.jp/ kokubu/ RIMS2006/proc.html. See also http://www.scholarpedia.org/article/Rotation_theory.

Department of Mathematics, National Tsing Hua University, Hsinchu, Taiwan

E-mail address: kchen@math.nthu.edu.tw

Department of Mathematics, University of Miami, Coral Gables, Florida 33124

Current address: Susquehanna International Group, 401 City Avenue, Suite 220, Bala Cynwyd, Pennsylvania 19004

E-mail address: xundong@math.miami.edu 